\title{
DISEÑO DE UNA ASIGNATURA SOBRE CONOCIMIENTOS DEL MERCADO PROFESIONAL DE LA TRADUCCIÓN EN EL MARCO DEL NUEVO GRADO DE TRADUCCIÓN E INTERPRETACIÓN Verónica Román Minguez. Universidad Autónoma de Madrid
}

\section{ABSTRACT}

The educational context of the study is based on the European Higher Education Area and in particular on the training of translators during the final year of the Translation and Interpretation degree studies at the Universidad Autónoma de Madrid.

The analysis applies to the learning and teaching of English-Spanish translation of legal and business documents in the private sector and is followed by providing a subject design included in the basic subjects of the review report of the Universidad Autónoma de Madrid Translation and Interpretation degree. This analysis aims to fill two gaps in the current curriculum of the UAM degree in Translation and Interpretation (Official State Gazette (Boletín Oficial de Estado) of 4/12/2002), that is to say, on the one hand, the absence of subjects that produce to the learner knowledge of the professional translation market and practice (certain peculiarities of the professional translation market, from the point of view of supply and demand of translation services and how these will affect the translation outcome, have been analysed in the study) and, on the other hand, the absence of subjects that produce specific knowledge of a given subject (basic knowledge of law, trade, economics and finance) that the new graduate will need to confront the translation of legal and business documents in the private sector.

KEYWORDS: translation studies, teaching translation, English-Spanish translation, legal translation, economic and financial translation, business documents.

\section{RESUMEN}

El contexto educativo del estudio se asienta en el Espacio Europeo de Educación Superior, y en concreto en el seno de la formación de traductores dentro del último curso de los estudios universitarios del grado en Traducción e Interpretación que se ofrecen en la Universidad Autónoma de Madrid. 
El análisis se aplica a la enseñanza-aprendizaje de la traducción (inglésespañol) de los documentos jurídico-empresariales en el ámbito privado y se materializa en el diseño de una asignatura que se incluyó en las materias básicas de la memoria de verificación del grado en Traducción e Interpretación de la Universidad Autónoma de Madrid. La propuesta pretende colmar dos lagunas que existen en el Plan de estudios actual de Licenciado en Traducción e Interpretación de la UAM (BOE 4/12/2002), esto es, por un lado, la ausencia de asignaturas que aporten conocimientos al alumno sobre el mercado profesional de la traducción y sobre la práctica profesional de la traducción (en el estudio se analizan algunas peculiaridades del mercado profesional de la traducción desde el punto de vista de la oferta y la demanda de servicios de traducción y cómo estas condicionan el resultado de la traducción) y, por otro, la ausencia de materias que aporten el conocimiento temático o enciclopédico (conocimientos básicos de derecho, comercio, economía y finanzas) que el alumno recién licenciado necesita para enfrentarse a la traducción de los documentos jurídico-empresariales en el ámbito privado.

PALABRAS CLAVE: estudios de traducción, didáctica de la traducción, traducción inglés-español, traducción jurídica, traducción económicofinanciera, documentos empresariales.

Antecedentes y justificación de nuestra propuesta

Contexto educativo

Algunas peculiaridades del mercado profesional de la traducción

Objetivos docentes

Contenidos docentes

Métodos docentes

Métodos de evaluación

Bibliografía

Anexos

\section{ANTECEDENTES Y RAZÓN DE SER DE NUESTRA PROPUESTA}

La Declaración de Bolonia de 1999 en el marco del Espacio Europeo de Educación Superior y la promulgación en nuestro país de la Ley Orgánica de Universidades (4/2007 de 12 de abril) ofrecen un nuevo modelo de enseñanzas que aporta una manera diferente de entender la Universidad y sus relaciones con la sociedad. Entre los objetivos de la Declaración de Bolonia se señala el de la adopción de un sistema de títulos fácilmente comprensibles y comparables a fin de promover la empleabilidad de los 
ciudadanos europeos y la competitividad del sistema de enseñanza superior europeo a escala internacional.

Asimismo, el espíritu de la Ley Orgánica de Universidades aprobada en España se sustenta en la idea de la implicación de las universidades en la respuesta a las demandas de la sociedad y el sistema productivo. Como se señala en la mencionada ley, las universidades, además de un motor para el avance del conocimiento, deben ser un motor para el desarrollo económico y social del país.

No nos detendremos aquí en los elogios o críticas que actualmente suscita el nuevo entorno en el que nos hayamos inmersos. Somos conscientes de que todos los cambios son difíciles, y este en concreto supone además un cambio importante de mentalidad.

Apuntaremos simplemente dos de los puntos fuertes que, en nuestra opinión, tiene el EEES y que, a nuestro parecer, contribuyen a acercar docencia y mercado profesional de la traducción:

a) Se trata de un modelo basado en el aprendizaje, esto es, el alumno debe "aprender a aprender". Esta educación a lo largo de toda la vida (lifelong learning) trae consigo un proceso permanente de interacción entre formación y trabajo, que en el caso del traductor profesional es absolutamente esencial.

b) En principio, confiamos en que el diseño de los nuevos grados se ajuste mucho más a la realidad del mercado profesional. Los egresados, al insertarse en el mercado laboral, podrán desempeñar puestos más acordes con los estudios cursados. El acercamiento de la formación ofrecida en la Universidad a la demanda de un mercado laboral cada vez más competitivo (esa es la realidad, nos guste o no) no nos parece que vaya en detrimento del avance del conocimiento. Tampoco pensamos que el ajuste del diseño de las materias de los nuevos grados a la realidad del mercado profesional implique hacer de la universidad un ente más prosaico.

Como señalan Bravo Utrera y Fidalgo González (2005), como docentes debemos trabajar en dos campos bien delimitados, el campo académicopedagógico y el campo profesional, orientado a contrastar la perspectiva del docente con la del profesional de la traducción y con la de los empresarios cuyas necesidades se relacionan con la traducción. Contemplamos la formación académica como una formación profesional que acerque el mundo académico al mundo empresarial y laboral.

A la luz de nuestra experiencia docente y profesional hemos constatado que el alumno termina sus estudios y presenta dificultades para hacer frente a muchas de las exigencias del mercado de la traducción profesional y que, además, desconoce las características y peculiaridades de este mercado. 
Asimismo, el alumno durante sus estudios no ha tenido oportunidad de analizar un abanico de textos amplio y representativo de la realidad profesional ni se ha enfrentado a las distintas circunstancias que pueden influir en un encargo de traducción por lo que desconoce como éstas pueden condicionar el resultado de la traducción.

En este sentido, en nuestra opinión, la competencia traductora que el alumno logra en el último curso de los estudios de Traducción e Interpretación se debería aproximar lo más posible a la que se exige en la práctica profesional de la traducción con el fin de que éste pueda responder a las exigencias del mercado profesional de la traducción de hoy en día.

Por esta razón, a nuestro parecer, la adquisición de las distintas destrezas traductoras en el aula debe realizarse en el marco de encargos reales de traducción y no con carácter previo a éstos.

Somos de la opinión de que el alumno debe tomar conciencia de que la tarea traductora no se puede abordar desde un único punto de vista y que éste debe tener en cuenta que las decisiones de traducción no son únicas y correctas en todo caso, sino que dependen del encargo de traducción en el que se encuentren inmersas. Como Señala Ortega Arjonilla (1999), debemos estar en guardia con respecto a los enfoques reduccionistas que pretenden considerar la traducción desde un único punto de vista.

De acuerdo con este objetivo, para nosotros la formación de traductores se fundamenta en la necesidad de situar el aprendizaje en el mundo real mediante experiencias auténticas. Por lo tanto, como señala Kirali (2000), el vehículo ideal para la adquisición de la competencia traductora en el aula será el encargo de traducción real.

En iguales términos se pronuncian Borja Albí y Monzó Nebot (2002) cuando señalan que resulta fundamental que en las clases de traducción se intente imitar el método de trabajo profesional y que, de esta manera, se inicie el proceso de aprendizaje con un encargo. El objetivo, como señalan estas autoras, consiste en motivar al estudiante mediante la propuesta de actividades que le acerquen a lo que será su futura vida profesional.

Ortega Arjonilla (1999) señala entre las condiciones que habrá de reunir un traductor cuando concluya su formación universitaria como traductor o intérprete lo siguiente: haber adquirido un método de traducción, unas estrategias, unas técnicas, etc., y conocer cómo funciona el mundo profesional de la traducción.

Señala Ortega Arjonilla que aunque la Universidad es muy dada a formar especialistas sin tener en cuenta lo que se les va a exigir una vez concluyan su formación universitaria, la juventud de los estudios de traducción e interpretación nos tendría que servir para establecer unos lazos estrechos con el mundo profesional de la traducción e interpretación por medio de la organización de prácticas profesionales tuteladas, el ofrecimiento de servicios 
de traducción e interpretación, etc. Esto, apunta Ortega Arjonilla, no va en detrimento de la libertad de cátedra del profesor ni de la formación académica que se debe exigir a un licenciado en Traducción e Interpretación, antes bien, permite al futuro licenciado conocer a fondo qué se le va a exigir como profesional una vez concluya su formación y qué sentido tiene saber de traducción (formación traductológica y adquisición de un método, de unas estrategias, de unas técnicas, etc.) para poder llegar a saber traducir profesionalmente (aplicando los conocimientos traductológicos y destrezas adquiridas en el proceso de formación universitaria).

\section{CONTEXTO EDUCATIVO}

El contexto educativo de nuestro estudio se asienta en el marco del Espacio Europeo de Educación Superior, y en concreto en el seno de la formación de traductores dentro del último curso de los estudios universitarios del grado en Traducción e Interpretación que se ofrecen en la Universidad Autónoma de Madrid1.

Nuestra propuesta se aplica a la enseñanza-aprendizaje de la traducción (inglés-español) de los documentos jurídico-empresariales en el ámbito privado y se materializa en el diseño de una asignatura que esperamos se incluya en las materias básicas del borrador de la memoria de verificación del grado en Traducción e Interpretación que se está elaborando por la Junta de la Facultad de Filosofía y Letras de la Universidad Autónoma de Madrid.

La mencionada asignatura se propuso como Curso Monográfico en Traducción para los estudios de segundo ciclo de la licenciatura en Traducción e Interpretación y se está impartiendo actualmente (curso académico 20082009) con tales características y como experiencia piloto ECTS.

Esta propuesta pretende colmar dos lagunas que existen en el Plan de estudios actual de Licenciado en Traducción e Interpretación de la UAM (BOE $4 / 12 / 2002)$, esto es, por un lado, la ausencia de asignaturas que aporten conocimientos al alumno sobre el mercado profesional de la traducción y sobre la práctica profesional de la traducción y, por otro, la ausencia de materias que aporten el conocimiento temático o enciclopédico que el alumno recién licenciado necesita para enfrentarse a la traducción de los documentos jurídico-empresariales en el ámbito privado.

En lo que respecta a la primera cuestión, hemos de señalar que el programa de prácticas externas desarrollado por el Decanato de la Facultad de

\footnotetext{
1 En la Universidad Autónoma de Madrid la licenciatura en Traducción e Interpretación se implantó en el año 2002. Desde entonces, la UAM es el único centro público que ofrece estos estudios en la Comunidad Autónoma de Madrid. Actualmente en la UAM se está trabajando en el diseño del nuevo grado en Traducción e Interpretación.
} 
Filosofía y Letras de la UAM está dando muy buenos resultados. Los alumnos que han realizado las prácticas resaltan la necesidad de poner en práctica este tipo de iniciativas con el fin de acercarse a la realidad profesional. Además, se ha solicitado la creación de una oficina de prácticas que enlace con las funciones del actual delegado del Decano para prácticas puesto que en los nuevos planes se prevé una presencia mucho más notable de las prácticas externas.

Creemos necesario, sin embargo, que el alumno adquiera en las aulas determinados conocimientos sobre el mercado profesional y la práctica profesional de la traducción con carácter previo al programa de prácticas y antes de su andadura profesional en una empresa determinada.

En relación con la segunda cuestión que apuntábamos, nos parece necesario diseñar una asignatura que permita al alumno adquirir conocimientos básicos de derecho, comercio, economía y finanzas, esto es, de las áreas del saber que coexisten de forma casi indisoluble en los documentos jurídicoempresariales.

En el actual plan de estudios de la UAM, como hemos señalado, no se recogen estos contenidos docentes. Además, con nuestra propuesta pretendemos proveer al alumno de las herramientas documentales y textuales necesarias para que aprenda a adquirir estos conocimientos por si mismo durante el último año de sus estudios y con el fin de que continúe haciéndolo a lo largo de su trayectoria profesional.

\section{ALGUNAS PECULIARIDADES DEL MERCADO PROFESIONAL DE LA TRADUCCIÓN}

En primer lugar, debemos señalar aquí, que entendemos la actividad objeto de nuestro análisis como una actividad ejercida por un profesional que presta servicios de traducción, ya sea dentro de una empresa (según un estudio de mercado llevado a cabo por la Agrupación de Centros Especializados en Traducción, ACT, en el año 2005 sólo un 15\% de las empresas tiene traductores en plantilla), en una agencia de traducciones o en calidad de profesional autónomo.

En lo que respecta a la demanda de servicios de traducción, nos interesa centrar nuestro análisis en la empresa privada. De este modo, dejaremos al margen los otros dos componentes de la demanda de servicios de traducción, a saber, el sector público y los particulares.

Centramos nuestro estudio en el ámbito privado ya que entendemos que el sector público presenta unas peculiaridades intrínsecas muy diferentes de las de la empresa privada. Además, a juzgar por nuestra experiencia profesional como traductores, consideramos que la demanda de servicios de traducción es 
más elevada en el sector privado que en el público. Por otra parte, el sector privado está mucho más sujeto a cambios que el sector público, es un sector mucho más dinámico e imprevisible y esto dificulta la tarea del traductor.

De esta forma, los textos objeto de nuestra propuesta serán los que emanen de las empresas o personas jurídicas en las que la propiedad del capital, la gestión, la toma de decisiones, y el control de las mismas se ejercen por agentes económicos privados y en las cuales el Estado no tiene ningún tipo de ingerencia.

En relación con la oferta de servicios de traducción, nos interesa la actividad del profesional autónomo, aunque haremos determinadas menciones a las agencias de traducción en calidad de intermediarios en el mercado de la traducción por la relación laboral que mantienen con el traductor autónomo. Dirigiremos nuestro interés hacia el profesional autónomo por dos razones:

a) como hemos señalado anteriormente, en España la cifra de traductores en plantilla es mucho más reducida que la de traductores autónomos.

b) el ejercicio de la profesión, tanto por traductores autónomos como por profesionales en plantilla o agencias de traducción, está sometido a las elevadas exigencias que impone el mercado y comporta claras peculiaridades y dificultades, pero además al traductor autónomo se le exigen otras habilidades o competencias a las que debe hacer frente por si mismo.

En nuestra opinión, una de las peculiaridades o dificultades que presenta el mercado de la traducción profesional en el ámbito de la empresa privada es que los clientes susceptibles de subcontratar una traducción son muy variados y de muy diversa naturaleza.

A la luz de nuestra experiencia profesional, entre los clientes-empresas que componen la demanda del mercado de la traducción profesional podemos destacar, sin ánimo exhaustivo, los siguientes:

a) bancos comerciales

b) bancos de inversión

c) entidades financieras

d) bufetes de abogados

e) empresas de consultoría

f) agencias de traducción

g) otras sociedades anónimas o limitadas con proyección internacional, con independencia de cual sea su actividad de negocio. Por citar algunas, agencias de comunicación, editoriales, empresas de telecomunicaciones, empresas de ingeniería, etc.

La amplitud y heterogeneidad de la demanda del mercado de la traducción profesional hace que la actividad que el traductor debe llevar a cabo sea una actividad compleja y difícil. 
Consideramos que las agencias de traducción componen tanto la oferta del mercado de la traducción como la demanda ya que ofrecen servicios de traducción a sus clientes y al mismo tiempo demandan servicios al traductor.

Podemos decir que el resto de clientes señalados anteriormente tienen un mismo denominador común, esto es, se trata de empresas multinacionales o que actúan en un ámbito transnacional y tienen la necesidad de solicitar una labor de mediación que les permita realizar de forma satisfactoria determinadas negociaciones o inversiones con personas físicas o jurídicas de nacionalidad extranjera.

Sin embargo, como ya hemos apuntado, este tipo de empresas son muy diferentes unas de otras y esto hace, salvo que el traductor trabaje siempre para el mismo tipo de cliente, que la tarea del traductor resulte muchas veces imprevisible.

Por lo señalado anteriormente, no nos resulta aventurado afirmar que en el mercado de la traducción profesional de la empresa privada rara vez existen dos encargos idénticos. Esto obliga al traductor a ser lo más versátil y polivalente posible para lograr adaptarse a las exigencias concretas de cada encargo y de cada cliente. Además, en términos generales, y al margen de determinadas salvedades en función del tipo de empresa de que se trate, el sector de la empresa privada es un sector moderno, dinámico y abierto en comparación con otros sectores. Esto hace que el traductor deba adaptarse para saber "comprender" los cambios que se producen en el seno de cada empresa privada.

De este modo, en nuestra opinión, existen determinados factores que pueden condicionar el resultado de la traducción, como por ejemplo, quién es el remitente del encargo (éste puede coincidir o no con el cliente y con el destinatario último de la traducción), el cliente (éste es uno de los factores que más condiciona el resultado de la traducción, pensemos por ejemplo en determinadas exigencias de calidad que pueda realizar éste o en algunas preferencias terminológicas que puedan condicionar los márgenes de libertad del traductor), el destinatario de la traducción (éste puede coincidir o no con el cliente), el plazo de entrega del TM (la mayoría de los encargos en este ámbito son urgentes), el enunciado del encargo (por ejemplo, la dificultad de interpretación de un posible encargo de traducción insuficiente o poco explícito, esto, desafortunadamente, sucede con bastante frecuencia y, en este sentido, los conocimientos prácticos y la experiencia acerca de la profesión de traductor pueden ayudar mucho al traductor), el formato del TO y el formato en el que el cliente quiere que entreguemos el TM (el formato condiciona el plazo y por lo tanto el resultado de la traducción), la tarifa que ofrece el cliente por la realización del trabajo (habrá que valorar si ésta compensa en relación con el tiempo y esfuerzo invertido), la exigencia por el cliente de herramientas informáticas (por ejemplo, Trados, Wordfast, Déjà $V u$, etc). Es importante que el 
traductor se asegure de que posee las mismas versiones de las herramientas informáticas que le solicita su cliente y que sabe cómo utilizarlas adecuadamente con el fin de ahorrar tiempo y evitar que supongan, más que una ayuda un lastre para su tarea), la valoración de hasta qué punto es responsable el traductor del producto final, (considerar si se trata de un trabajo de traducción en el que el traductor tiene que asumir la revisión y autoedición de su trabajo o existe alguien más que vaya a tomar decisiones al respecto antes de publicar dicha traducción), las fuentes de documentación al alcance del traductor para la realización del trabajo (por citar algunas, textos paralelos, glosarios y documentación utilizados para ese mismo cliente, diccionarios especializados bilingües y monolingües, bases de datos terminológicas, foros de traducción), etc.

A nuestro parecer, es importante que el traductor adquiera competencia profesional, esto es, que aprenda a desarrollar las habilidades necesarias para poder moverse satisfactoriamente en el mundo profesional, por ejemplo, como ya hemos apuntado, la de respetar los plazos, valorar si es capaz de dominar las herramientas informáticas que le exige el cliente, reconocer si un trabajo supera el volumen de palabras que se es capaz de traducir por día, etc.

En este sentido, como ya hemos señalado anteriormente, el encargo o proyecto de traducción en el aula supone una motivación para el estudiante porque entraña una propuesta de actividades que le acercan a lo que será su futura vida profesional.

Esta necesidad de recrear situaciones reales de la práctica profesional sólo se puede calibrar si los docentes mantenemos un contacto cercano y actualizado con el mundo de la traducción profesional o la realidad del mercado laboral con el fin, en última instancia, de adaptar los planes de estudios a las necesidades del mercado laboral y las necesidades de los estudiantes. Al hablar de la competencia traductora Kiraly (2000: 26) afirma lo siguiente:

Translator trainers would surely agree that translator competence should be the primary goal of our translator education programmes. It goes without saying that our institutional programmes should be producing graduates that the community of professional translators as well as the clients of translators' services would judge to be competent language mediators.

Para Kelly (2002: 14) la competencia traductora "es la macrocompetencia que constituye el conjunto de capacidades, destrezas, conocimientos e incluso actitudes que reúnen los traductores profesionales y que intervienen en la traducción como actividad experta".

De lo señalado anteriormente se desprende la conclusión de que la calidad de las traducciones está muy relacionada, más que con criterios o 
estándares objetivos con el tipo de cliente y otras circunstancias en las que pueda circunscribirse cada encargo. Como ya hemos señalado, el cliente es uno de los factores que más condiciona el resultado de una traducción.

Suscribimos la opinión de Mayoral cuando dice que una traducción es eficaz cuando surte el efecto para el que ha sido encargada y que para ello debe cumplir con determinados requisitos de calidad, forma y plazos establecidos tanto por el cliente como por el destinatario final; debe ajustarse a sus expectativas (Mayoral Asensio, 1997).

En este sentido, Schaffner (1998) considera que la calidad es algo que debe negociarse entre el cliente y el traductor.

Asimismo, apunta Mayoral Asensio, la calidad no es una cuestión de sí o no (bueno o malo) sino de grado y que guarda relación con las diferentes exigencias de diferentes tipos de encargos. Los conceptos de aceptabilidad y adecuación en el mundo profesional pueden ser absolutamente contradictorios con el concepto académico de la calidad. Otros conceptos como rendimiento, rentabilidad, productividad, cumplimiento de plazos ${ }^{2}$, etc. (originados en la práctica profesional) también chocan de frente con el concepto académico de calidad (Mayoral Asensio, 2000).

En relación con los factores que pueden incidir en el rendimiento de un traductor

Zabalbeascoa (2000) apunta los siguientes: competencia traductora, conocimiento de las variedades concretas de las lenguas del texto de partida, TP, y del TM, las convenciones y los contextos socioculturales, discursos, géneros, el léxico, el iniciador y sus expectativas, los receptores de la traducción y sus expectativas y valores, los temas tratados, contexto laboral (existencia y viabilidad de un encargo más o menos explícito, diccionarios, hojas de estilo, bases de datos, textos paralelos y cualquier otro tipo de ayuda documental, personal y material, y, además el plazo de entrega), experiencia previa en la traducción (redacción o lectura de textos semejantes en algún aspecto), incentivo y motivación, colaboración y corrección por parte de otras personas cualificadas.

Para el mencionado autor el rendimiento y, por lo tanto, la evaluación de la actuación de un traductor, depende de la competencia traductora, pero este factor no es el único parámetro que puede explicar el resultado final de una traducción. Una mayor competencia traductora es el factor que permite que un traductor rinda mejor que otro siempre y cuando todas las variables contextuales y las condiciones materiales sean las mismas para ambos y que ambos tengan el mismo entusiasmo y conocimiento de los temas tratados y del tipo de traducción requerido. Un traductor puede compensar en alguna medida

\footnotetext{
2 El énfasis es de Mayoral Asensio (2000).
} 
una falta de competencia y mejorar su rendimiento si logra trabajar en condiciones óptimas y muy motivado.

En iguales términos se pronuncia el estudio realizado en 2005 por la ACT cuando señala que el sector de la oferta de la traducción se enfrenta a un reto en la implantación y explotación de las nuevas tecnologías para la traducción como es el de la oportunidad de dar respuesta a las demandas de productividad y calidad lingüística.

La complejidad de la tarea traductora de la que hablamos se acentúa además, en nuestra opinión, por el hecho de que en el mundo de la empresa privada las necesidades de los clientes que mencionábamos anteriormente se tienen que cubrir en un espacio de tiempo muy corto. Este apremio se pone de manifiesto en los plazos de los encargos de traducción que son muchas veces muy difíciles de cumplir.

Las empresas privadas realizan múltiples actividades que comportan inversiones o negociaciones relacionadas con su actividad de negocio y que deben hacerse la mayoría de las veces con urgencia.

Asimismo, las agencias de traducción y los bufetes de abogados que actúan a menudo en calidad de intermediarios tienen que hacer frente a la competencia del mercado y, a fin de no perder clientes, prometen o aceptan fechas que muy a menudo son totalmente inaceptables. Estas empresas necesitan con urgencia el texto objeto de encargo ya que tienen, a su vez, un plazo muy ajustado respecto de su cliente y además necesitan unos días de margen para procesar internamente el texto antes de enviárselo al destinatario final.

Como señala Mayoral Asensio, decir que una traducción debe ser rentable significa que debe proporcionar una plusvalía a todos los que invierten dinero o trabajo en la misma, desde el cliente al mismo traductor, y para ello debe realizarse en el mínimo tiempo que permitan las condiciones de eficacia (Mayoral Asensio, 1997b).

Más aún, a nuestro parecer, la urgencia por la que se caracteriza el mercado de la traducción profesional en el mundo de la empresa privada se trasluce también en la dificultad que tiene muchas veces el traductor para recibir feedback por parte del cliente.

Como ya hemos apuntado anteriormente, el cliente con necesidades de traducción está muy desbordado, el mundo empresarial es vertiginoso y la persona que solicita un servicio de traducción no tiene apenas tiempo para solucionar las dudas o problemas del traductor. Esto hace que la responsabilidad del trabajo final recaiga muchas veces únicamente sobre el traductor (algo menos en el caso de que el traductor trabaje con un intermediario en el mercado como por ejemplo una agencia de traducciones, un bufete de abogados, una empresa consultora o un banco de inversión que a su vez presten servicios a su cliente). 
Por lo tanto, el traductor deberá estar acostumbrado a solucionar problemas, tomar decisiones o asumir los riesgos intrínsecos a esta compleja tarea con el fin de entregar a su cliente textos definitivos que permitan a éste satisfacer sus necesidades de comunicación de forma inmediata. En este sentido, por ejemplo, las notas de traducción a pie de página, la utilización de dos términos en vez de uno para dejar con el fin de que el cliente realice la elección, son prácticas poco recomendables en el estresado mundo de la traducción profesional de la empresa privada.

Además, a la complejidad de la labor traductora desempeñada en el mercado profesional de la empresa privada debemos añadir la complejidad que comporta toda actividad traductora por ser la traducción una actividad multidimensional. El traductor deberá tener claro que para traducir en este ámbito de especialidad, al igual que en otros, no basta con la mera competencia lingüística.

Como señalan Bravo Utrera y Fidalgo González (2005), la traducción es una habilidad que se desarrolla a partir de conocimientos lingüísticos, estrategias y técnicas de traducción, documentación y terminología, conocimientos socioeconómicos, históricos y culturales, dominio de las más modernas herramientas informáticas, entre otros, requiere, además, de información actualizada acerca de los movimientos del mercado laboral en el que trabajarán los futuros traductores.

Ortega Arjonilla se pronuncia en términos similares en una entrevista publicada para los alumnos en la página web de la Universidad Jaume I de Castellón cuando apunta que el traductor "se hace" y no "nace", aunque se tengan aptitudes más o menos evidentes para el aprendizaje de idiomas. Ortega Arjonilla considera que es cuestión de aptitudes y de "voluntad", al 50\%, y recomienda el trabajo en equipo para poder sobrevivir en el mercado de la traducción especializada: "es necesario contar con traductores-revisores con "tablas" para aprender lo que tiene de "buen hacer" y de "oficio" este trabajo nuestro.

Si bien esta claro que, al igual que en toda actividad traductora el traductor tendrá que adquirir determinadas subcompetencias traductoras (terminológicas, temáticas, documentales, textuales, de dominio de las nuevas tecnologías, etc.), en lo que respecta a la competencia temática necesaria para traducir textos en este ámbito de especialidad, hemos de señalar que, en nuestra opinión, ésta es amplísima.

El área del saber en la que se insertan los distintos documentos susceptibles de traducción en el ámbito de la empresa privada está formada por varias disciplinas y por esta razón resulta más conveniente hablar de "las áreas del saber" en las que este ámbito se circunscribe. En nuestra opinión, en los documentos jurídico-empresariales del sector privado aparecen conceptos y terminología propios del derecho, economía, comercio y finanzas, así como 
otros propios de otros ámbitos de especialidad en función del objeto social de cada una de las empresas que demandan servicios de traducción.

Teniendo en cuenta lo anterior el traductor en este campo deberá estar permanentemente formándose para intentar abarcar el "mar de conocimientos" en el que se circunscribe la actividad traductora en este ámbito. En nuestra opinión es importante que el traductor sea consciente de que deberá hacerlo sin "morir en el intento" ya que resulta imposible adquirir la competencia temática del experto en las diferentes subáreas que aparecen en este ámbito de especialidad. Por esta razón, la competencia temática del traductor de textos jurídico-empresariales deberá ser instrumental y utilitaria, esto es, al servicio de la traducción.

La actividad de la traducción profesional en el ámbito de la empresa privada es una actividad económica, desarrollada en un mercado, el de la traducción, en el que un profesional, el traductor, presta sus servicios a otra persona jurídica, en este caso una empresa privada, a cambio de unos honorarios o una remuneración.

No olvidemos que el mercado de la traducción es un mercado muy competitivo y a veces la competencia del mercado obliga a aplicar tarifas muy bajas. El hecho de ser una profesión que cualquiera puede ejercer sin necesidad de acreditar ningún tipo de experiencia ni formación, combinado con la creación relativamente reciente de la licenciatura, hace que sea una de las profesiones con un índice de intrusismo más elevado. Además, existe naturalmente una pugna entre los traductores que se inician en la profesión y que deben introducirse en el mercado y hay cierta tendencia entre ellos a ofrecer sus servicios a precios más bajos que los que tienen los traductores ya consolidados.

Indudablemente, los honorarios que el traductor percibe por su trabajo repercuten en su motivación profesional y condicionan el resultado de la traducción. Por ejemplo, muchas veces existe el riesgo de sacrificar la calidad para lograr que las tarifas bajas resulten rentables (por ejemplo, se traduce a ritmo vertiginoso, no se dedica tiempo a la labor de documentación, no se revisa detenidamente el trabajo final, etc.).

A nuestro parecer, si no se va a hacer un buen trabajo porque la tarifa no es rentable a veces es preferible decir "no" a un cliente. El problema es que cuando se está empezando es difícil rechazar trabajos. Además, la actividad autónoma de la traducción lleva aneja una serie de gastos fijos para el ejercicio de la profesión que hay que cubrir para no salir empeñado, tales como el pago de la cuota de autónomos todos los meses (alrededor de 244,35 euros), honorarios a la gestoría si el traductor no se aventura a llevar su propia contabilidad, gastos relacionados con la adquisición de fuentes documentales como diccionarios, libros, gastos derivados de la utilización de herramientas informáticas como la línea adsl, fax, página web personal, programas 
informáticos, cuentas de correo electrónico, gastos relacionados con el asociacionismo (según datos de la ACT, el nivel de asociacionismo es bajo entre las empresas de traducción, posiblemente inferior al 15\%, mientras que los traductores autónomos tienen mayor tendencia a pertenecer a una asociación del sector, se calcula que en 2005 había aproximadamente un 44\% de profesionales asociados), etc.

Por todo lo señalado anteriormente el mercado profesional de la traducción de la empresa privada es, en nuestra opinión, un mercado exigente con los traductores que subcontrata porque exige que éstos tengan las competencias necesarias para llevar a cabo su labor de traducción, esto es, las competencias intrínsecas a toda labor traductora (que como ya hemos señalado van más allá de la mera competencia lingüística, esto es, competencia temática, especialización, competencias documentales, competencias textuales, dominio de herramientas informáticas y de las nuevas tecnologías, etc.).

Pero además se le exigen al traductor otras competencias relacionadas con las características del mercado de la traducción profesional en este ámbito como son, entre otras, cumplimiento de los ajustados plazos de los encargos de traducción o capacidad para trabajar bajo presión, capacidad de adaptarse a las distintas exigencias de cada encargo de traducción en función del cliente de que se trate, habilidades sociales o capacidad de trabajo en grupo en el caso de trabajar con revisores o colegas, y otras dotes como por ejemplo la capacidad para soportar una vida solitaria o un flujo de trabajo no constante.

Otra dificultad con la que cuenta el traductor autónomo que se lanza al mercado es la de conseguir clientes. Es indudable que el traductor además de todas las dotes señaladas anteriormente necesita tener determinadas habilidades comerciales. Cuando el traductor comienza su andadura profesional le resulta muy difícil entrar en el mercado y suele hacerlo de la mano de las agencias de traducción, teniendo en cuenta que muchas de ellas pagarán unos honorarios muy bajos al traductor éste tiene que valerse de sus contactos y hacer una buena labor comercial que le permita forjar su primera cartera de clientes.

\section{OBJETIVOS DOCENTES}

Partiendo de la necesidad de acercar docencia y práctica profesional de la traducción señalamos los objetivos docentes que perseguimos con el diseño de nuestra asignatura:

1) Adquisición de conocimientos sobre el mercado profesional de la traducción del mundo de la empresa privada y sobre las peculiaridades y dificultades de la actividad traductora desarrollada en ese ámbito. 
2) Aprender a diferenciar y conocer los distintos tipos textuales del mundo de la empresa privada (documentos jurídico-empresariales, económicos, financieros y comerciales) y ser capaz de reconocer e interpretar diferentes niveles de significado, registros y estilos en los textos.

3) Capacidad de análisis de las distintas circunstancias que pueden concurrir en un encargo de traducción del mundo de la empresa privada y cómo éstas condicionan el resultado de la traducción.

4) Dominar los distintos recursos y herramientas existentes para comprender la terminología y conceptos de los documentos del mundo de los negocios.

5) Adquisición de conocimiento temático en relación con las áreas del saber que confluyen en la traducción de los documentos del ámbito jurídicoempresarial (comprensión de los conceptos básicos de derecho, economía, comercio y finanzas que se encierran tras la terminología propia de los mencionados textos).

6) Capacidad de análisis y búsqueda de soluciones para las distintas dificultades de traducción (inglés-español) que puedan aparecer en los distintos tipos textuales del mundo de la empresa privada (documentos jurídico-empresariales, económicos, financieros y comerciales).

\section{CONTENIDOS DOCENTES}

Los estudios de Traducción e Interpretación tienen un marcado componente práctico, por lo que la docencia en este campo requiere una conjunción cuidadosa entre los fundamentos teóricos de la materia que se imparte y el desarrollo y seguimiento de las tareas asignadas a los alumnos.

Por la razón apuntada anteriormente, al diseñar nuestra asignatura hemos clasificado los contenidos docentes en contenidos teóricos y prácticos, si bien somos conscientes de que, en una disciplina como la Traducción, la línea divisoria entre unos y otros resulta difusa.

Contenidos teóricos:

1) Características del mercado de la traducción profesional del mundo de la empresa privada. El traductor del mundo de la empresa privada: competencia traductora y práctica profesional.

2) Características del inglés y del español jurídico, económico, financiero y comercial.

3) Los tipos textuales del mundo de la empresa privada (documentos jurídicoempresariales, económicos, financieros y comerciales).

4) Los encargos de traducción del mundo de la empresa privada. Análisis de los distintos factores en los que se puede circunscribir un encargo de traducción en este ámbito. 
5) Fuentes documentales para la comprensión de la terminología y conceptos que aparecen en los documentos del mundo de la empresa privada en lengua inglesa y española: análisis de la bibliografía facilitada.

6) Breve introducción al derecho: fuentes del derecho, ramas del derecho y otros conceptos jurídicos básicos.

7) Conceptos jurídicos básicos aplicables a la traducción de los principales tipos textuales del mundo de la empresa privada.

8) Nociones básicas de derecho mercantil: Los principales documentos de las sociedades mercantiles.

9) Conceptos económicos básicos aplicables a la traducción de los documentos del mundo de la empresa privada.

10) Conceptos y terminología financieros, bancarios y bursátiles básicos aplicables a la traducción de los documentos del mundo de la empresa privada.

11) Conceptos básicos sobre cuentas anuales. La terminología de las cuentas anuales (balances, cuentas de pérdidas y ganancias y memorias anuales).

12) Salidas profesionales y "supervivencia" en el mercado profesional de la traducción del mundo de la empresa privada.

13) Testimonios de distintos profesionales del ámbito de la empresa privada: traductores en plantilla, gestores de proyectos de agencias de traducciones, abogados, economistas, consultores, etc.

Contenidos prácticos:

-Ejercicios de análisis de posibles encargos de traducción y de las distintas circunstancias que pueden confluir en ellos (en grupo y dentro del aula)

-Ejercicios de análisis del discurso inglés y español (análisis de textos origen y de traducciones para valorar convenciones textuales, detección de falsos amigos, préstamos y calcos) (dentro del aula)

-Ejercicios para la adquisición de competencia textual (elaboración de un banco de documentos en lengua inglesa y española del área del saber de los negocios), (autoaprendizaje, fuera del aula)

-Ejercicios de documentación para la adquisición de conocimiento temático sobre derecho, economía y finanzas (utilización de todo tipo de fuentes) (autoaprendizaje fuera del aula)

-Ejercicios de traducción a la vista (individuales y dentro del aula)

-Encargos simulados de traducción (individuales y en grupo) (autoaprendizaje, fuera del aula)

-Ejercicios de revisión de traducciones (individuales y en grupo) (dentro y fuera del aula)

-Tutorías, individuales o en grupo, con el fin de resolver dudas de traducción, aclarar cuestiones teóricas y realizar diferentes tareas didácticas.

-Lecturas obligatorias con el fin de que el alumno adquiera conocimiento temático. 


\section{MÉTODOS DOCENTES}

Para el logro de nuestros objetivos docentes al principio de cada clase damos al alumno unas breves nociones teóricas (siempre utilitarias y al servicio de la traducción) que versarán sobre la actividad traductora en el mundo de la empresa privada, los diferentes encargos de traducción en ese ámbito, análisis de las características del inglés y español de los negocios, fuentes documentales aplicables a la traducción y conocimiento temático mínimo del área del saber del derecho, economía y finanzas.

A continuación complementamos la teoría con varios ejercicios prácticos para reforzar o poner en práctica lo aprendido. En los ejercicios prácticos se fomentará el trabajo, la toma de decisiones y la participación de los alumnos. Los ejercicios que presentamos son de dos tipos:

a) los realizados en clase, en grupo, entre los alumnos y la profesora, basados en el aprendizaje cooperativo.

b) los realizados fuera del aula en los que el alumno deberá desarrollar el autoaprendizaje

\section{MÉTODO DE EVALUACIÓN}

El método de evaluación de la asignatura se inspira en estos tres principios:

a) método de evaluación continua, con el fin de que el alumno "no se lo juegue todo a una carta".

b) técnicas de aprendizaje cooperativo y aprendizaje basado en proyectos.

c) sistema de incentivos con el fin de estimular al alumno en su aprendizaje.

A lo largo del cuatrimestre los alumnos deberán realizar varias pruebas de evaluación:

1. Prueba sobre los conocimientos teóricos adquiridos (conocimientos tanto de las peculiaridades del discurso de los negocios como de los conceptos básicos del área del saber del derecho, economía y finanzas). Esta prueba será individual y se realizará en horas lectivas. Cuenta un $20 \%$ para la calificación final de la asignatura.

2. Prueba de demostración de competencias documentales y textuales. Se realizará en grupo y fuera del aula. Cuenta un $20 \%$ para la calificación final.

3. 10 lecturas obligatorias que se realizarán fuera del aula y sobre las que el profesor evaluará una serie de entregas individuales:

-hoja de respuestas a preguntas teóricas

-realización de resúmenes o extractos de las lecturas 
-hoja de comentarios, dudas y reflexiones del alumno

De acuerdo con el sistema de incentivos, la realización por parte del alumno de las 10 entregas supondrá un 5\% respecto de la calificación final. La calificación obtenida como media aritmética de las 10 entregas contará un 15\% sobre la calificación final.

4. Traducción individual de un texto jurídico-económico. Cuenta un $20 \%$ sobre la calificación final.

5. Encargo simulado de traducción. Se realizará en grupo y cuenta un $10 \%$ sobre la calificación final de la asignatura.

6. Revisión de una traducción. Se realizará en grupo y cuenta un $10 \%$ sobre la calificación final de la asignatura.

\section{BIBLIOGRAFÍA}

Act (Agrupación de Centros Especializados en Traducción) (2005): Estudio de situación del mercado español de servicios profesionales de traducción. http://isg.urv.es/library/papers/nunez mercado.ppt\#1

Borja Albí y Monzó Nebot, E. (2002a): "Aplicación de los métodos de aprendizaje cooperativo a la enseñanza de la traducción jurídica: cuaderno de bitácora", en Edutec, Congreso internacional de tecnología, educación y desarrollo sostenible. Murcia: Universidad de Murcia.

Bravo Utrera y FidAlgo GonZÁlez, L. (2005): “Aplicaciones didácticas de los resultados de una investigación de mercado en la enseñanza de la traducción escrita", en IV Jornadas sobre la Formación y Profesión del Traductor e Intérprete. Madrid: Publicaciones de la Universidad Europea de Madrid. Disponible en URL:

www.uem.es/web/fil/invest/publicaciones/web/autores/bravo utrera art.htm. [Fecha de consulta: 3 de mayo de 2006]

KELLY, D. (2002): "Un modelo de competencia traductora: bases para el diseño curricular", en Puentes 1. Granada: Comares.

KIRALI, D.C. (2000): A Social Constructivist Approach to Translator Education. Manchester: St. Jerome.

Ley Orgánica de Universidades (4/2007 de 12 de abril) http://www.boe.es/boe/dias/2007/04/13/pdfs/A16241-16260.pdf

MAYORAL AsENSIO, R. (1997): "La traducción especializada como operación de documentación”, en Sendebar. Granada: Universidad de Granada.

Mayoral Asensio, R. (2000): ¿Son los estudios de traducción una ciencia? Disponible en

URL: http://www.gitrad.uji.es/common/articles/Mayoral1.pdf. [Fecha de consulta: 11 de julio de 2007] 
Ortega Arjonilla, E. (1999): Proyecto docente y de investigación. Docencia en traducción especializada francés-español, español-francés (B). Textos científico-técnicos. Plaza de profesor titular de Universidad del área de Traducción e Interpretación de la Universidad de Málaga. 2 volúmenes. Inédito. Málaga: Universidad de Málaga.

SCHAFFNER, CH. (ed.) (1998): Translation and Quality. Manchester: St. Jerome.

ZABALBEASCOA, P. (2000): La didáctica de la traducción: desarrollo de la competencia traductora. Barcelona: Universidad Pompeu Fabra. [Disponible en URL: http://cvc.cervantes.es/obref/aproximaciones/zabalbeascoa.htm\#10.

Fecha de consulta: 3 de marzo de 2005].

\section{ANEXOS}

Presentamos a título meramente ilustrativo tres ejercicios prácticos de los diseñados para la asignatura propuesta:

EJERCICIO PRÁCTICO N4 (AUTOAPRENDIZAJE)

Utiliza las fuentes documentales que te presentamos en el apartado "Referencias de Consulta Básicas" de la Guía Docente de la asignatura y cualesquiera otras fuentes a las que puedas tener acceso en Internet y encuentra los siguientes documentos en lengua inglesa y española:

\section{Employment contract}

Minutes of a Board of Directors' Meeting

Balance Sheet

Balance

Estatutos sociales

Carta del Presidente

No olvides apuntar la fuente de la que has extraído cada uno de los documentos con el fin de comprobar su fiabilidad.

Los textos que debes encontrar pueden ser tanto textos reales como modelos. Hint: puedes utilizar el recurso a expertos para obtener textos reales (si este fuera el caso, no olvides apuntar el nombre, cargo y empresa a la que pertenece el experto). También puedes acceder a los modelos de documentos en inglés y en español que te presentamos en el apartado "Referencias de Consulta Básicas" de la Guía Docente de la asignatura. 
Asegúrate de archivar los textos que encuentres para que puedas utilizarlos como textos paralelos para traducciones que podamos realizar en esta asignatura o para encargos ulteriores que te puedan surgir en el ejercicio de la profesión.

\section{EJERCICIO PRÁCTICO No5 (AUTOAPRENDIZAJE)}

Echa un vistazo a los equivalentes de traducción que acuñamos en el Ejercicio 2 y utiliza fuentes fiables en Internet con el fin de encontrar al menos un TO en lengua inglesa y su correspondiente TM en lengua española.

Hint: Los apartados relativos a la información para inversores y accionistas de las páginas web de algunas de las empresas que forman el IBEX 35 en España suelen tener publicada la documentación en versión bilingüe.

\section{EJERCICIO PRÁCTICO Nº (EN EL AULA)}

En grupos de 10 alumnos se evalúan los documentos presentados por cada uno de los alumnos al grupo en virtud de los siguientes criterios:

-Idoneidad y pertinencia de los documentos de acuerdo con las instrucciones de los Ejercicios 4 y 5 anteriores.

-Fiabilidad de las fuentes de las que se han extraído los textos.

Se juntan todos los documentos seleccionados y se separa el resto de documentos. El objetivo consiste en compartir información entre los estudiantes y en elaborar un mini banco documental que pueda ser utilizado por todos los miembros del grupo. 